\title{
Rotation of stars in NGC $6134^{\star}$
}

\section{A comparison of $\delta$ Scuti stars and non-variable stars}

\author{
M. B. Rasmussen ${ }^{1}$, H.Bruntt ${ }^{1}$, S. Frandsen ${ }^{1}$, E. Paunzen ${ }^{2}$, and H. M. Maitzen ${ }^{2}$
}

1 Institute for Physics and Astronomy, University of Aarhus, Bygn. 520, 8000 Aarhus C, Denmark

2 Institut für Astronomie der Universität Wien, Türkenschanzstrasse 17, 1180 Wien, Austria

Received 14 February 2002 / Accepted 24 April 2002

\begin{abstract}
We present results of spectroscopic observations of selected stars in the southern open cluster NGC 6134. We have determined the rotational velocities of the six known $\delta$ Scuti stars in NGC 6134 as well as several other non-variable stars with similar colour temperature in order to investigate if $v \sin i$ and variability is somehow connected: we find no such correlation. We also compare the distribution of $v \sin i$ of $\delta$ Scuti stars and non-variable stars with four other well-studied open clusters to look for any systematic behaviour, but we find no conclusive evidence for $v \sin i$ and variability to be connected. We have also used the spectra to carry out an abundance analysis of the $\delta$ Scuti stars in NGC 6134 to confirm the high metal content of the cluster. We find $[\mathrm{Fe} / \mathrm{H}]=+0.38 \pm 0.05$ which is in agreement with the result obtained from Strömgren photometry. We also present $\Delta a$ photometry of the cluster, but we find no chemical peculiar stars based on this index.
\end{abstract}

Key words. stars: oscillations - stars: variable: $\delta$ Scuti - stars: rotation - open clusters and associations: individual: NGC 6134 , NGC 3680

\section{Introduction}

The open cluster NGC 6134 has six confirmed $\delta$ Scuti star members (Frandsen et al. 1996) of which five are multiperiodic. High quality Strömgren photometry has been carried out by Bruntt et al. (1999), except that the $c_{1}$ index (requiring $u$ images) could not be obtained with the CCD detector. This paper describes a continuation of the earlier programs. We use spectroscopy and $\Delta a$-photometry (Maitzen 1976; Maitzen \& Vogt 1983) to obtain more parameters and additional information about the stars in the cluster. These parameters are needed for the modeling and interpretation of the oscillations in the $\delta$ Scuti stars. Here we will derive rotational velocities $(v \sin i)$ for variable and non-variable stars and carry out abundance analysis for the $\delta$ Scuti stars.

In principle, with the combination of the information from the frequencies of the $\delta$ Scuti stars and the constraints imposed on the variables by being members of an open cluster we will be able to make a very powerful test of stellar evolution. The analysis of $\delta$ Scuti stars is very complicated in stars that are fast rotators, so it is important to locate slow rotators before extensive campaigns are started to obtain oscillation spectra with very low noise levels, leading to the detection of many oscillation frequencies. For example, the open cluster Praesepe has

Send offprint requests to: S. Frandsen, e-mail: srf@ifa.au.dk

* Based in part on observations obtained at the European Southern Observatory at La Silla and UTSO-Las Campanas, Chile.
$14 \delta$ Scuti stars and all but one rotate fast $\left(v \sin i>100 \mathrm{~km} \mathrm{~s}^{-1}\right)$ (Rodríguez et al. 2000). This has complicated the determination of the stellar parameters considerably and made the identification of the observed frequencies with radial or non-radial modes very difficult (Pérez Hernández et al. 1999; Kjeldsen et al. 1998). The situation is not quite as bad for the Pleiades and Hyades clusters where a few slowly rotating $\delta$ Scuti stars are found.

The nature of the excitation mechanism in $\delta$ Scuti stars is an unsolved problem - especially the understanding of which among the many possible modes predicted from models have obserable amplitudes. We have looked for systematic differences between variable and non-variable stars. When the variables were discovered, a large set of non-variable stars (amplitude $<1 \mathrm{mmag}$ ) were identified. It is therefore possible to find pairs of stars, variable and non-variable (twins), with similar photometric parameters. The $\delta$ Scuti stars are located in the instability strip and intrinsically unstable due to the opacity mechanism; but the $\delta$ Scuti stars have modes that do not seem to follow any regular pattern. Many stars in the instability strip do not vary at all. Perhaps the explanation is the difference in observables like $[\mathrm{Fe} / \mathrm{H}]$ and $v \sin i$ among $\delta$ Scuti stars and non-variable stars in the instability strip. We will examine this hypothesis by comparing variable and non-variable stars with approximately the same colour-temperature and evolutionary state. 


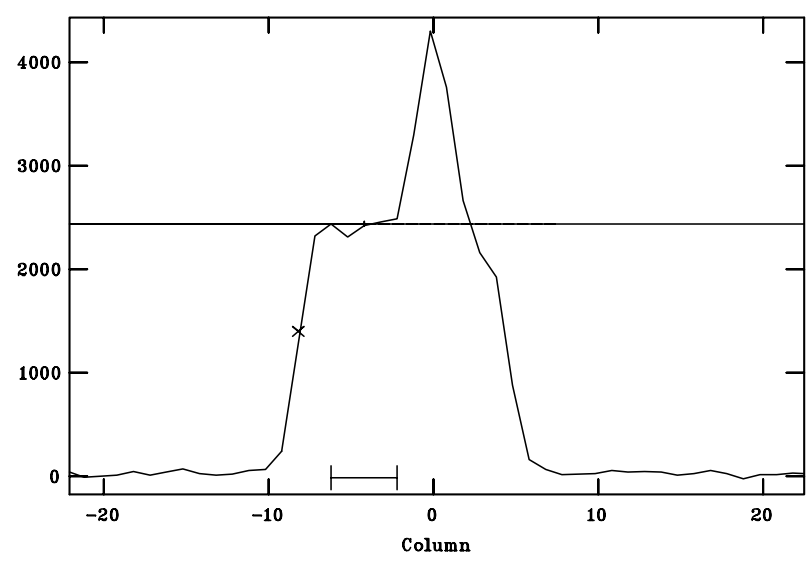

(a) The non-stellar background signal is very high in this case.

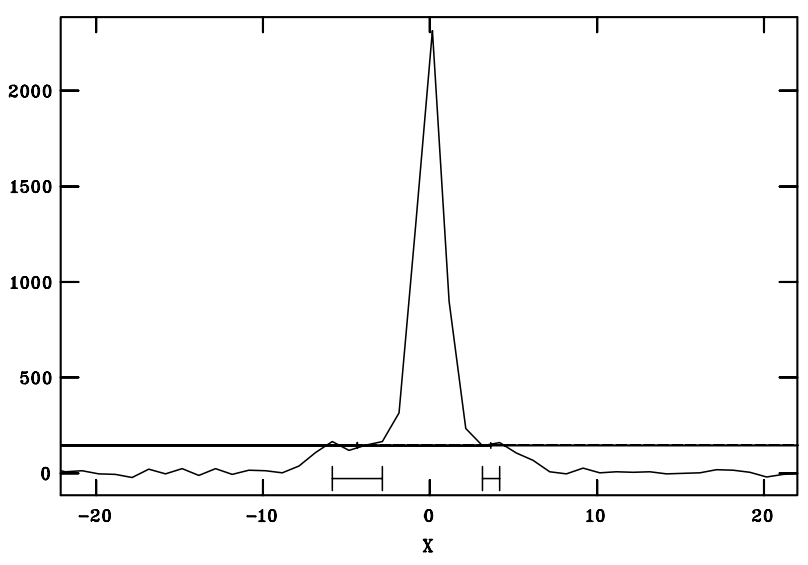

(b) For this star the background level is still significant but not dominating.

Fig. 1. Expanded plot of the cross section of an order for two different stars. The $x$-axis measures the distance from the center of the order in pixels. The horizontal bars in the lower part of each plot show how we have set the background regions in IRAF during manual editing of the apertures when using the task apall. In a) the high background signal indicates that the Moon is nearby.

Finally, we try to confirm the metallicity determined from Strömgren photometry (Bruntt et al. 1999) and to locate possible Am or Ap-type stars among the $\delta$ Scuti stars. We will do abundance analysis of the $\delta$ Scuti stars and we also investigate any possible chemical peculiarity with $\Delta a$ photometry. It seems that the diffusion leading to chemically peculiar stars is coupled with slow rotation and seems to exclude variability (Kurtz 2000). But since NGC 6134 is a metal rich cluster, with a large number of A-type stars with high values of the Strömgren $m_{1}$ index - also in case of the $\delta$ Scuti stars - it would be interesting if some of these are CP-type stars.

The observations are described in Sect. 2 and in Sect. 3 we describe the reduction of the spectra. The calibration of the stellar parameters is given in Sect. 4 and in Sect. 5 we determine the rotational velocities. The abundance analysis is carried out in Sect. 6. The data reduction and results of the $\Delta a$ photometry is given in Sect. 7. In Sect. 8 we gather in information on each of the known $\delta$ Scuti stars and in Sect. 9 we give a general discussion of our results before giving our conclusions in Sect. 10.

\section{The observations}

Spectra were obtained with the DFOSC on the Danish $1.54 \mathrm{~m}$ telescope at ESO, La Silla during the period May 24th to June 2nd, 1999. The Echelle mode was used, employing the combination of grism \#9 cross dispersed by grism \#11. The detector was a Loral/Lesser C1W7 CCD and was read out via amplifier $\mathrm{A}$ at high gain and binning by a factor 2 in the $x$-direction perpendicular to dispersion of grism \#9 was performed. The spectral resolution is $R=4300$ and the wavelength range is 3000-11000 А.

The resolution of the DFOSC is too small for velocity measurements and abundance studies, but it is a very efficient instrument. The $\delta$ Scuti stars are faint $(V \simeq 12-13)$ and a large telescope is needed to carry out high resolution studies. Flexure problems prevented precise measurements of radial velocities which could have been used to study membership of the cluster and binarity.

Two out of eight nights were lost due to bad weather. The total number of spectra collected for the rest of the observing period was 66 for stars in NGC 6134: typically 3-5 spectra for each of the six $\delta$ Scuti stars and 2-3 spectra of nine selected "twin" stars. The twin stars were selected from the Strömgren photometry, i.e. we chose stars with similar values of the $b-y$ index and $V$ magnitude. Except for the $\delta$ Scuti stars \#853 and \#906 (the evolved ones), the differences between the photometric values of the variable and its twin were rather small, i.e. $|\Delta(b-y)|<0.004$ and $|\Delta(V)|<0.55$ which corresponds to $\left|\Delta\left(T_{\text {eff }}\right)\right|<280 \mathrm{~K}$ and $|\Delta(\log g)|<0.3$. For the two evolved $\delta$ Scuti stars the differences were somewhat larger with $\Delta\left(T_{\text {eff }}\right) \simeq-430 \mathrm{~K}$ and $\Delta(\log g) \simeq-0.5$, i.e. both $\delta$ Scuti stars have higher values of $T_{\text {eff }}$ and $\log g$ compared to their twins.

For calibration purposes we also obtained a total of $37 \mathrm{spec}-$ tra of 10 stars in NGC 3680 and 28 spectra of bright reference field stars - the 13 of which were of HD 118646. The exposure times were $900-1200 \mathrm{~s}$ for stars in NGC 6134, at least $1200 \mathrm{~s}$ for stars in NGC 3680, and 40-300 s for the field stars.

The $\Delta a$-photometry was obtained in a program including four other open clusters (see Bayer et al. 2000). The Bochum $61 \mathrm{~cm}$ telescope at ESO and the Helen-Sawyer-Hogg $61 \mathrm{~cm}$ telescope at UTSO (Las Campanas) were used with CCD cameras with a field of view around 4 arcmin. A total of 32 frames were obtained over 6 nights. The detailed data analysis will be published elsewhere (Paunzen \& Maitzen 2002).

\section{Data reduction}

The reduction of the Echelle spectra was complicated by several factors. The observations took place during bright time. 

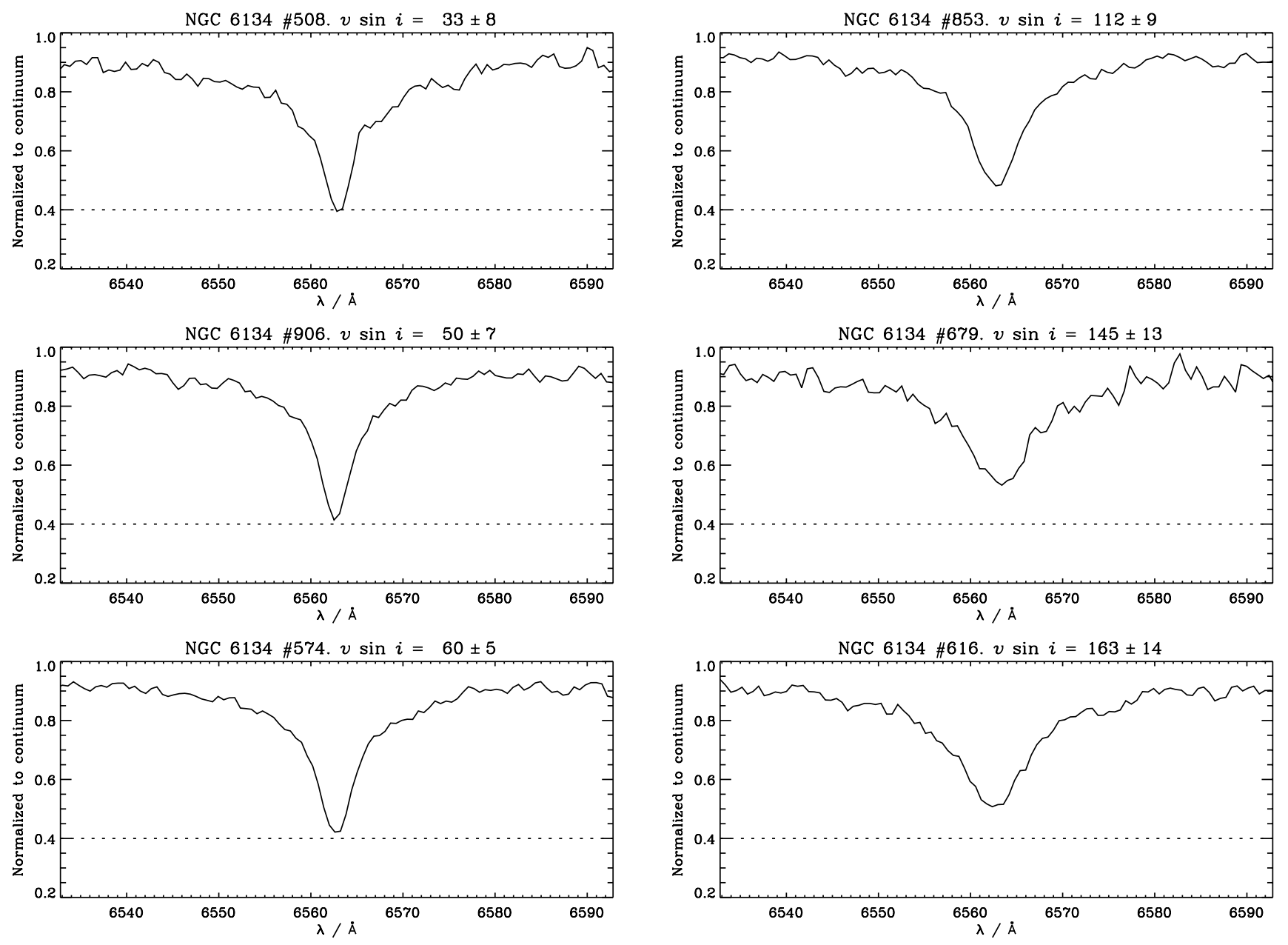

Fig. 2. $\mathrm{H} \alpha$ of the six $\delta$ Scuti stars plotted for comparison. The dashed line at 0.4 is just a visual aid.

Hence, scattered Moon light results in a relatively high sky background level, especially for the fainter targets. Two examples are shown in Fig. 1.

The large flexures in the DFOSC make it necessary to shift the mask used to extract the 2D spectra from one frame to the next. This also makes it difficult to combine spectra of the same target to increase $S / N$. Often the $v \sin i$ was measured for each frame and the average $v \sin i$ is computed for the individual exposures. In order to calibrate the wavelength a large number of Th-Ar spectra were needed, one before and after each exposure. We decided to give up attempts to measure radial velocities at the precision needed to determine membership $\left(\sigma_{V} \leq 1 \mathrm{~km} \mathrm{~s}^{-1}\right)$ and instead to use the telescope time for obtaining spectra of stars.

Due to the long exposure times night sky emission lines appear in the spectra and this makes the background determination more cumbersome. They do contribute in a positive sense by providing a sort of wavelength calibration.

The stellar image tends to drift on the slit during and between exposures. The illumination of the entrance slit is therefore changing all the time, making the instrumental response vary in time.
The extraction of the spectra from the CCD frames was done using partly IRAF and partly IDL routines developed to overcome the insufficiencies of IRAF.

\subsection{Spectral resolution}

The region around the $\mathrm{H} \alpha$ line is shown in Fig. 2 for the six $\delta$ Scuti stars. The nominal resolution of the spectrograph using grism \#9 is $R=4300$ with a $1^{\prime \prime}$. 0 slit. We were using a slit of $1^{\prime \prime}$. 5 corresponding to a resolution of $R=2900$, but on some nights the seeing was at or below 1 .' 0 , effectively increasing the resolution up to $R \simeq 5000$. This is the maximum $R$ as it corresponds to 2 pixels on the detector.

\subsection{Flexures of the spectrograph}

The effect of the flexures is displayed in Fig. 3, which mainly illustrate our inability to compensate for the flexures in the DFOSC and the variation in the illumination of the slit. Wavelength shifts of the order of $1 \AA$ appear as a result. 


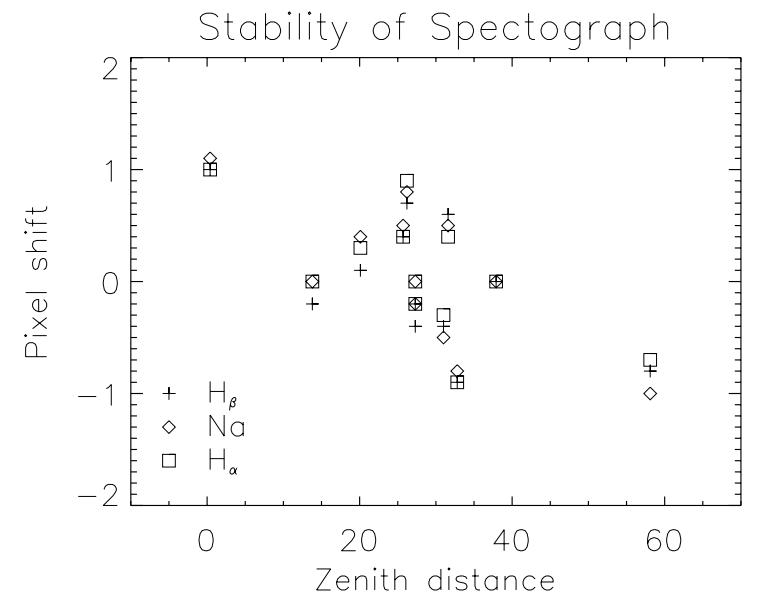

Fig. 3. Variations of the position of selected spectral lines for the reference star HD 118646 from night to night, sorted by zenith distance. $\mathrm{H} \beta$ $(\lambda 4261 \AA)$ is located in the fifth aperture. The Na doublet $(\lambda 5889 \AA)$ is in aperture \#8. $\mathrm{H} \alpha(\lambda 6563 \AA)$ is in aperture \#9. Estimated uncertainties of the line positions: \pm 0.1 pix.

\section{Calibration of stellar parameters}

The basic cluster parameters are given in Table 1 and are based on existing data. In order to be able to make precise comparisons of the results from the spectra and the results from the Strömgren photometry presented by Bruntt et al. (1999), a recalibration of the photometry has been done. We have used the TEMPLOGG code by Rogers (1995) which automatically selects the most appropriate photometric calibration based on the Strömgren indices. The program then interpolates the dereddened indices in the atmospheric ATLAs6 model grid by Kurucz (1979) and determines the fundamental atmospheric parameters of the star, i.e. $T_{\text {eff }}, \log g$, and $[\mathrm{Fe} / \mathrm{H}]$. We note that the photometric calibrations considered by TEMPLOGG are not the bare results from the ATLAs6 grids, but rather an experimental calibration - i.e. the color grids are transformed so as to match well-calibrated fundamental stars before the calibration is made.

We found that the $T_{\text {eff }}$ values are systematically a few hundred degrees lower than what is given in Bruntt et al. (1999). The effect of this change in $T_{\text {eff }}$ is a change in the age and distance to the clusters. The distances found by Bruntt et al. (1999) decrease by $25 \%$ and the ages increase by $0.1 \mathrm{Gyr}$ (cf. Table 1 ).

When comparing synthetic spectra to the observations to derive rotational velocities we confirmed that the spectra were in better agreement with the somewhat lower values of $T_{\text {eff }}$.

For the $\delta$ Scuti star \#906 (and star \#919, cf. Table 5) the $m_{1}$ index is outside the range of the templogg program; the metallicity of this star was derived from the calibration of Atype stars by Smalley (1993).

A few stars have evolved far off the main sequence and are outside the range of the TEMPLOGG code. The $\log g$ was then derived by comparison with stars with the same $T_{\text {eff }}$ but lying on the main sequence by using the difference in $V$ magnitude and assuming the same bolometric correction.

The parameters that were derived for the $\delta$ Scuti stars and the known variable blue straggler in NGC 6134 are listed in
Table 1. Basic parameters for the observed open clusters.

\begin{tabular}{lrr}
\hline \hline Parameter & \multicolumn{1}{c}{ NGC 6134 } & \multicolumn{1}{c}{ NGC 3680 } \\
\hline Reddening $^{a}, E(b-y)$ & $0.263 \pm 0.004$ & $0.048 \pm 0.011$ \\
Metallicity $^{a},[\mathrm{Fe} / \mathrm{H}]$ & $+0.28 \pm 0.02$ & $0.09 \pm 0.02$ \\
Cluster distance $^{b}, d$ & $1.05 \pm 0.06 \mathrm{kpc}$ & $0.83 \pm 0.05 \mathrm{kpc}$ \\
Cluster age $^{c}, A$ & $0.79 \pm 0.10 \mathrm{Gyr}$ & $1.58 \pm 0.15 \mathrm{Gyr}$ \\
Right ascension, $\alpha^{\mathrm{h}}$ & $13^{\mathrm{h}} 27^{\mathrm{m}} 42^{\mathrm{s}}$ & $11^{\mathrm{h}} 25^{\mathrm{m}} 42^{\mathrm{s}}$ \\
Declination, $\delta$ & $-49^{\circ} 09^{\mathrm{m}} 00^{\mathrm{s}}$ & $-43^{\circ} 15^{\mathrm{m}} 00^{\mathrm{s}}$ \\
\hline
\end{tabular}

${ }^{a}$ From Bruntt et al. (1999).

${ }^{b}$ From Bruntt et al. (1999); distance decreased by $25 \%$.

${ }^{c}$ From Bruntt et al. (1999); age decreased by 0.1 Gyr.

Table 2. The average metallicity for NGC 6134 is $[\mathrm{Fe} / \mathrm{H}]=$ $+0.28 \pm 0.02$ (Bruntt et al. 1999); the quoted error is the standard deviation of the mean for 50 F-type stars, and does not include any possible systematic errors. We estimate the error on the metallicity to be at least 0.10 dex which corresponds to an error on the zero point of the $m_{1}$ index of $10 \mathrm{mmag}$. Hence, we will use $[\mathrm{Fe} / \mathrm{H}]_{\text {phot }}=+0.28 \pm 0.10$ from this point.

\section{Rotational velocities}

The rotational velocities were derived by comparing the observed spectrum with a synthetic spectrum where temperature, $\log g$, and metallicity were determined from the Strömgren indices. The synthetic spectrum $F_{\lambda \text {,synth }}$ was convolved with the instrumental response $P_{\lambda \text {,instr }}$ and the rotational broadening function $G_{\lambda}(v \sin i)$, thus:

$F_{\lambda, \mathrm{obs}}=F_{\lambda, \mathrm{synth}} \otimes P_{\lambda, \text { instr }} \otimes G_{\lambda}(v \sin i)$.

We then determine $v \sin i$ by minimizing the residuals when subtracting the observed and convolved synthetic spectrum. In the following we will discuss in detail the technique we have used and the results.

\subsection{The synthetic spectra}

The synthetic spectra were supplied by Werner W. Weiss, Vienna, calculated using a modified version of ATLAs9 (Kupka 1996) and the line lists from the vald database (Kupka et al. 1999). The grid was a coarse grid with the following properties:

- $T_{\text {eff }} \in[5800,8000] \mathrm{K}$ with $\Delta T=250 \mathrm{~K}$;

- $\log g \in[3.80,4.50]$ with $\Delta \log g=0.5$;

- $[\mathrm{Fe} / \mathrm{H}] \in[0.00,0.50]$ with $\Delta[\mathrm{Fe} / \mathrm{H}]=0.5$.

The wavelength range is $4000-8000 \AA$ and the resolution is $0.1 \AA$ A. For a given star a spectrum was interpolated in this grid to the calibrated parameters using the following weights:

$W_{i}=\left(1-\frac{\Delta\left(T_{\mathrm{eff}}\right)_{i}}{250}\right)\left(1-\frac{\Delta(\log g)_{i}}{0.5}\right)\left(1-\frac{\Delta([\mathrm{Fe} / \mathrm{H}])_{i}}{0.5}\right)$. 
Table 2. Identification numbers and Strömgren indices from Bruntt et al. (1999) as well as the derived astrophysical parameters for the six $\delta$ Scuti stars and the blue straggler (star \#647) in NGC 6134. The $c_{1}$ index was determined from the $c_{0}$ indices (Bruntt et al. 1999). The last three columns contain data from the program TEMPLOGG (Rogers 1995). The standard errors on $T_{\text {eff }}, \log g$, and [Fe/H] are $200 \mathrm{~K}, 0.2$ dex, and 0.2 dex respectively.

\begin{tabular}{ccccccccccc}
\hline \hline $\mathrm{ID}_{3}$ & $V$ & $(b-y)$ & $m_{1}$ & $c_{1}$ & $\beta$ & $E(b-y)$ & $(b-y)_{0}$ & $T_{\text {eff }}$ & $\log g$ & {$[\mathrm{Fe} / \mathrm{H}]$} \\
\hline 508 & 13.548 & 0.422 & 0.159 & 0.859 & 2.784 & 0.256 & 0.159 & 7540 & 3.85 & 0.59 \\
574 & 12.452 & 0.461 & 0.104 & 0.902 & 2.738 & 0.271 & 0.198 & 7110 & 3.47 & 0.23 \\
616 & 13.179 & 0.415 & 0.136 & 0.889 & 2.778 & 0.252 & 0.152 & 7470 & 3.79 & 0.36 \\
647 & 11.673 & 0.277 & 0.209 & & 2.898 & & 0.014 & & & \\
679 & 13.547 & 0.424 & 0.144 & 0.806 & 2.758 & 0.236 & 0.161 & 7320 & 3.86 & 0.46 \\
853 & 11.973 & 0.477 & 0.120 & 0.967 & 2.742 & 0.264 & 0.214 & 6660 & 3.32 & 0.49 \\
906 & 12.266 & 0.486 & 0.181 & 0.802 & 2.698 & 0.227 & 0.223 & 6780 & 3.47 & 1.01 \\
\hline
\end{tabular}

\subsection{The instrumental profile}

The seeing was generally good and smaller than the slit width. The mean instrumental profile was estimated by a Gaussian profile. The width of the Gaussian was determined by calculating $v \sin i$ for a slowly rotating star HD 118646 with a well determined $v \sin i=35.3 \pm 1.7 \mathrm{~km} \mathrm{~s}^{-1}$ (mean of the results from Glebocki \& Stawikowski 2000). The seeing for a given spectrum was estimated by measuring the width of the spectrum perpendicular to the dispersion direction. This width determines the width of the Gaussian instrumental profile and gives consistent results for the reference star HD 118646. Further tests were possible using a few other reference stars with known $v \sin i$.

The stars in NGC 3680 are slow rotators due to the relatively higher age of the cluster (compared to NGC 6134) and the spectral type of the observed stars (F-type stars), i.e. magnetic braking has had time to reduce the rotation. Unfortunately the observed stars around the turn-off region are quite faint and the spectra have poor $S / N$. Due to the wide instrumental profile, $v \sin i$ values below $25 \mathrm{~km} \mathrm{~s}^{-1}$ are not considered: the value is just quoted as being below $25 \mathrm{~km} \mathrm{~s}^{-1}$.

\subsection{Rotational broadening}

We follow Gray (1992) and use for $G_{\lambda}(v \sin i)$ :

$G=\frac{2(1-\epsilon)\left[1-\left(\Delta \lambda / \Delta \lambda_{\mathrm{L}}\right)^{2}\right]^{\frac{1}{2}}+\frac{1}{2} \pi \epsilon\left[1-\left(\Delta \lambda / \Delta \lambda_{\mathrm{L}}\right)^{2}\right]}{\pi \Delta \lambda_{\mathrm{L}}(1-\epsilon / 3)}$,

where

$\Delta \lambda=v=(\lambda / c) x \Omega \sin i$,

and

$\Delta \lambda_{\mathrm{L}}=(\lambda / c) v \sin i$.

A limb-darkening law is used in the form:

$I_{\mathrm{c}} / I_{\mathrm{c}}^{0}=1-\epsilon+\epsilon \cdot \cos \theta$,

where $\theta$ is the angle formed by the line of sight and the direction of the emerging flux. $I_{\mathrm{c}}^{0}$ represents the intensity at the stellar disk center. The linear limb-darkening coefficient is chosen to have a value of $\epsilon=0.5$. Using a constant value has a negligible effect on the results.

\subsection{Spectral lines used for determination of $v \sin i$}

The rotational velocity is determined from a small set of individual spectral lines. For the slowly rotating stars we have the choice of using a set of narrow metal lines and the strong hydrogen line profiles, which are wide but have deep core absorption. For the faster rotators the metal lines become very shallow and blended. The influence of the continuum fit begins to dominate the accuracy and only the hydrogen lines give good results.

In Table 3 we give the list of the metal lines that were used to measure $v \sin i$ in HD 118646.

The strong Na doublet is perturbed by lines from intervening interstellar lines and cannot be used.

\subsection{Rotation of stars in NGC 6134}

The rotational velocities for the $\delta$ Scuti stars and their twins in NGC 6134 are given in Table 4. Note that a twin star can be a twin for more than one $\delta$ Scuti star and some twins appear more than once in Table 4. A $\delta$ Scuti star can also have more than one twin. There are seven twins for six $\delta$ Scuti stars. Star \#647 is not a $\delta$ Scuti star but a variable blue straggler, and it is included here for completeness.

It seems that there is no evidence for any difference in terms of rotational velocity between $\delta$ Scuti stars and stable stars. This is not a strong statement due to the small number of stars involved, but it is still interesting.

Our result that the $v \sin i$ of the $\delta$ Scuti stars are evenly distributed from 30 to $180 \mathrm{~km} \mathrm{~s}^{-1}$ is in good agreement with Solano \& Fernley (1997). We find the same distribution for the non-variable stars but here Solano \& Fernley find a larger spread in rotational velocity. The most likely reason for this is that we have a small sample of stars compared to Solano \& Fernley.

\subsection{Rotation of other stars}

Among the 10 stars we observed in NGC 3680 most of them have $V \simeq 14-15$ and were thus fainter than our targets in NGC 6134 where the range among the 15 observed stars is $V \simeq 12-13$. Thus the $S / N$ was much lower for the stars in NGC 3680 and the determination of the $v \sin i$ is more uncertain. In summary we find that our sample of stars in 
Table 3. A list of 21 metallic lines in HD 118646 with sufficient width and isolation to be usable for determination of $v \sin i$. The table is divided by horizontal lines which correspond to the location of the three orders of the spectrograph that we have used. The line position in the spectrum has not been corrected for heliocentric motion. The equivalent widths $(E W)$ depend heavily on the determination of the continuum.

\begin{tabular}{|c|c|c|c|}
\hline$\lambda^{\text {synth }} / \AA$ & $\lambda^{\mathrm{obs}} / \AA$ & Line(s) & $E W / \mathrm{m} \AA$ \\
\hline 4957.489 & 4958.29 & $\mathrm{Fe}_{\mathrm{I}} / \mathrm{Fe}_{\mathrm{I}}$ & 293 \\
\hline 5183.608 & 5184.82 & $\operatorname{Mg}_{\mathrm{I}}(\lambda 5183.604)$ & 495 \\
\hline 5226.913 & 5227.99 & Ti п & 512 \\
\hline 5328.290 & 5329.59 & $\mathrm{Fe}_{\mathrm{I}} / \mathrm{Cr}_{\mathrm{I}} / \mathrm{Fe}_{\mathrm{I}}$ & 434 \\
\hline \multirow[t]{4}{*}{5446.915} & 5447.51 & $\mathrm{Fe}_{\mathrm{I}}(\lambda 5446.916)$ & 275 \\
\hline & 5476.65 & $\mathrm{Fe}_{\mathrm{I} / \mathrm{Fe}} \mathrm{I} / \mathrm{Ni}_{\mathrm{I}}$ & 354 \\
\hline & 5447.51 & $\mathrm{Fe}_{\mathrm{I}}(\lambda 5446.916)$ & 275 \\
\hline & 5477.87 & $\mathrm{Fe}_{\mathrm{I} / \mathrm{Fe}} \mathrm{I} / \mathrm{Ni}_{\mathrm{I}}$ & 288 \\
\hline 5528.416 & 5529.18 & $\mathrm{Sc}$ пI $/ \mathrm{Mg}_{\text {I }}$ & 284 \\
\hline 5615.564 & 5616.69 & $\mathrm{Fe}_{\mathrm{I}} / \mathrm{Fe}_{\mathrm{I}}$ & 236 \\
\hline 5763.676 & 5754.90 & $\mathrm{Fe}_{\mathrm{I}} / \mathrm{Fe}_{\mathrm{I}} / \mathrm{Si}_{\mathrm{I}}$ & 210 \\
\hline 5762.953 & 5764.10 & $\mathrm{Fe}$ I/Si I & 190 \\
\hline 6102.764 & 6103.95 & $\mathrm{Fe}_{\mathrm{I} / \mathrm{Ca}} \mathrm{I} / \mathrm{Fe} \mathrm{I} / \mathrm{Fe}$ II & 222 \\
\hline 6122.235 & 6123.48 & Са I $(\lambda 6122.217)$ & 159 \\
\hline \multirow[t]{3}{*}{6136.750} & 6138.35 & $\mathrm{Fe}_{\mathrm{I}} / \mathrm{Fe}_{\mathrm{I}} / \mathrm{Fe}_{\mathrm{I}}$ & 254 \\
\hline & 6142.96 & $\mathrm{Ba}$ II/Si I & 140 \\
\hline & 6163.49 & $\mathrm{Ca} / \mathrm{Ca}$ I & 273 \\
\hline \multirow[t]{5}{*}{6169.349} & 6170.93 & $\mathrm{Ca} / \mathrm{Ca}_{\mathrm{I}} / \mathrm{Fe}_{\mathrm{I}}$ & 200 \\
\hline & 6279.44 & $\mathrm{Sc}_{\mathrm{II}} / \mathrm{Fe}_{\mathrm{I}}$ & 449 \\
\hline & 6457.38 & $\mathrm{Ca}$ I/Fe II/Si I & 204 \\
\hline & 6496.52 & $\mathrm{Ca} \mathrm{I} / \mathrm{Fe} \mathrm{I} / \mathrm{Fe} \mathrm{I} /$ & \\
\hline & - & $\mathrm{Fe} \mathrm{I} / \mathrm{Ba}$ II/Ca I & 646 \\
\hline
\end{tabular}

Table 4. Mean values of $v \sin i\left(\right.$ in $\mathrm{km} \mathrm{s}^{-1}$ ) for the variable stars and their twins in this study. Star \#647 is a blue straggler. The errors quoted are the standard deviation of the mean.

\begin{tabular}{cccc}
\hline \hline$\delta$ Scuti star & $v \sin i$ & Twins & $v \sin i$ \\
\hline 508 & $33+8$ & 218 & $35+7$ \\
574 & $60+5$ & 754 & $90+16$ \\
& & 919 & $88+6$ \\
& & 969 & $157+15$ \\
616 & $163+14$ & 689 & $90+11$ \\
& & 865 & $115+14$ \\
647 & $120+25$ & & \\
679 & $145+13$ & 218 & $35+7$ \\
853 & $112+9$ & 969 & $157+15$ \\
906 & $50+7$ & 830 & $105+18$ \\
\hline
\end{tabular}

NGC 3680 are all slow rotators and only an upper limit of $25 \mathrm{~km} \mathrm{~s}^{-1}$ could be determined - a limit which is due to the resolution of the spectrograph.

We also observed several reference stars. Most of them have $v \sin i$ below $25 \mathrm{~km} \mathrm{~s}^{-1}$ and the exceptions are: for HD 118646 we find $v \sin i=28.5 \pm 1.6$, HD 132475 has $v \sin i=35 \pm 10$, and for HD 155458 we find $v \sin i=105 \pm 20$. For HD 118646 the mean of three measurements listed in Glebocki \& Stawikowski (2000) is $v \sin i=35.3 \pm 1.7 \mathrm{~km} \mathrm{~s}^{-1}$ which is in rough agreement with what we find. The value of $v \sin i=11 \pm 6 \mathrm{~km} \mathrm{~s}^{-1}$ for HD 132475 quoted in Glebocki \&
Stawikowski (2000) is from Stetson (1983). This is calculated using a method which depends on the calibration of a single star (HD 24450) which has a poorly known value of $v \sin i$ (see p. 1362 in Stetson 1983) and this may be the cause for the disagreement with our result result of $v \sin i=35 \pm 10 \mathrm{~km} \mathrm{~s}^{-1}$.

The method was also tested on two spectra obtained with different instruments. A spectrum from the ELODIE spectrograph at $R \approx 48000$ of the $\delta$ Scuti star HD 49434 was analysed. We found $v \sin i=70 \mathrm{~km} \mathrm{~s}^{-1}$ using $\mathrm{H} \alpha$ and $v \sin i=80 \mathrm{~km} \mathrm{~s}^{-1}$ using $\mathrm{H}_{\beta}$. This is in good agreement with the value of $v \sin i=$ $84 \mathrm{~km} \mathrm{~s}^{-1}$ measured by Bruntt et al. (2002).

To summarize, our results for the field stars HD 118646 and HD 49434 agree with independent determinations, and confirm the validity of the $v \sin i$ produced by our technique.

\section{Abundances}

The average metallicity of NGC 6134 was determined from the $m_{1}$ index of the F-type stars by Bruntt et al. (1999), i.e. $[\mathrm{Fe} / \mathrm{H}]_{\text {phot }}=+0.28 \pm 0.10$. In addition Bruntt et al. (1999) found that several of the A-type stars have a very high $m_{1}$ index, indicative of a high metallicity. The reddening corrected $m_{0}$ index is shown versus the temperature sensitive $\mathrm{H}_{\beta}$ index in Fig. 5 The turn-off stars lie around $\beta=2.77 \pm 0.02$ and temperature increases to the left.

For two of the $\delta$ Scuti stars a quite high $m_{1}$ index has been measured (star number \#508 and \#906, cf. Table 2). The high abundances of metals in A-type stars is thought to be an effect of diffusion in the upper layers present in slowly rotating stars with stable envelopes, which would tend to decrease also the content of Helium in the driving layers and make the star stable. Interestingly, high metallicity and variability is suspected to be exclusive phenomena (Kurtz 2000).

In order to confirm this we decided to test the $[\mathrm{Fe} / \mathrm{H}]$ results from the Strömgren photometry by doing abundance analysis by using the low resolution spectra obtained to measure $v \sin i$. We calculated the atmosphere models with a modified version of the ATLAS9 code (Kupka 1996), and extracted line lists from valD (Kupka et al. 1999). We have developed software called vwa for semi-automatic abundance analysis which is described in Bruntt et al. (2002): vwA automatically selects lines that suffer from the the least amount of blending. For a region around each selected line the synthetic spectrum is calculated for different abundances of the element forming the line until the equivalent width of the observed and synthetic spectrum match. When this is done, each fitted line is inspected visually - and the fit may either be accepted, rejected or improved manually.

The abundance analysis did not produce reliable results for all the stars. For most of the non-variable stars only two spectra were obtained, hence the $S / N$ is small and the errors on the derived abundances are large. Since we obtained more spectra of the $\delta$ Scuti stars in NGC 6134 we were able to make the best abundance determination for some of these stars. The exceptions are the $\delta$ Scuti stars \#616, \#647, and the blue straggler \#853 for which the combination of high $v \sin i$ and low $S / N$ made the abundance analysis impossible. 

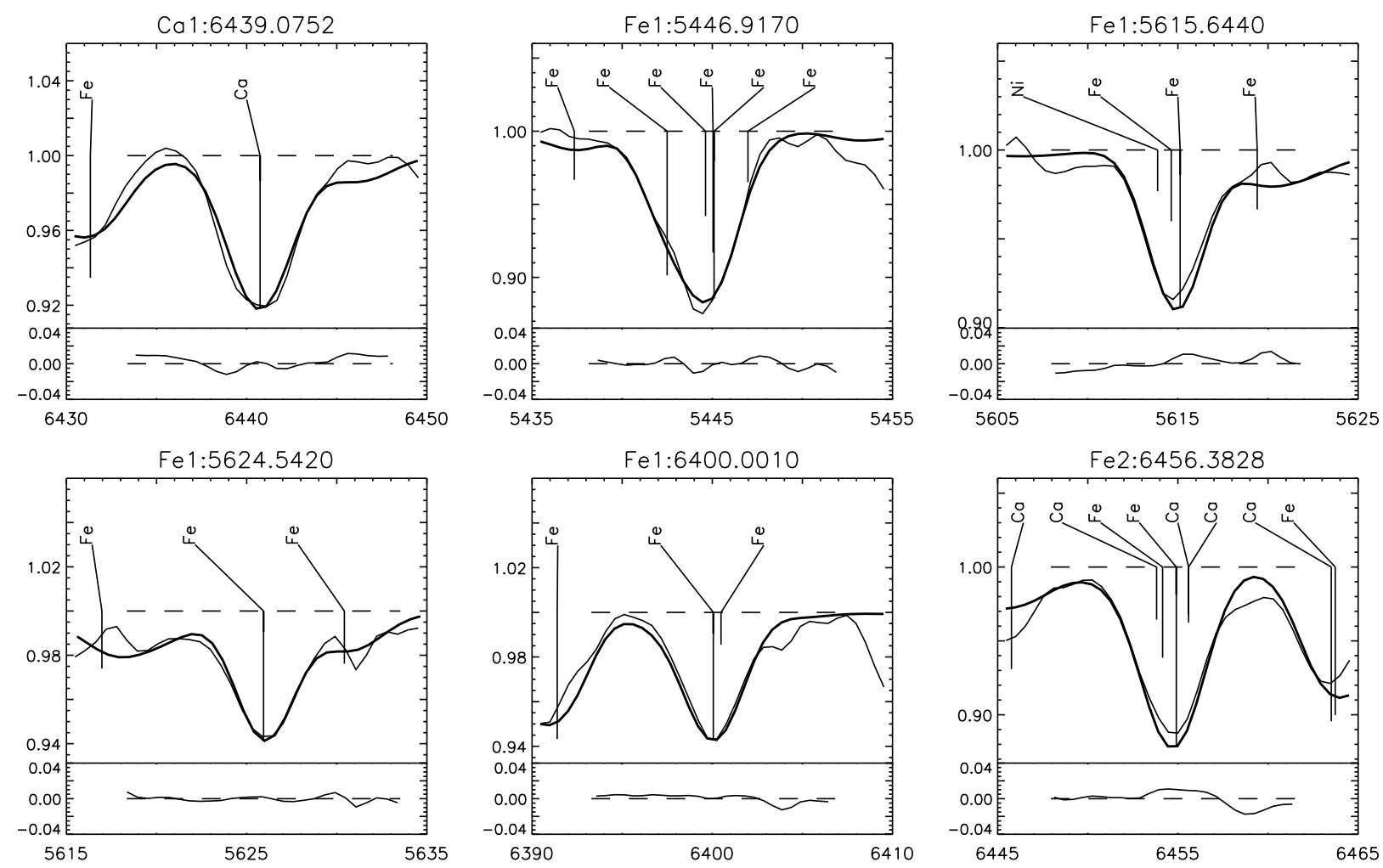

Fig. 4. The observed (thin line) and fitted synthetic spectrum (thick line) for six lines for the $\delta$ Scuti star \#574. The lower portion of each plot shows the relative difference of the observed and synthetic spectrum. The position of the fitted line and the deepest neighbouring lines are marked by vertical lines. The length of these lines are scaled with the theoretical line depth of the fitted line.

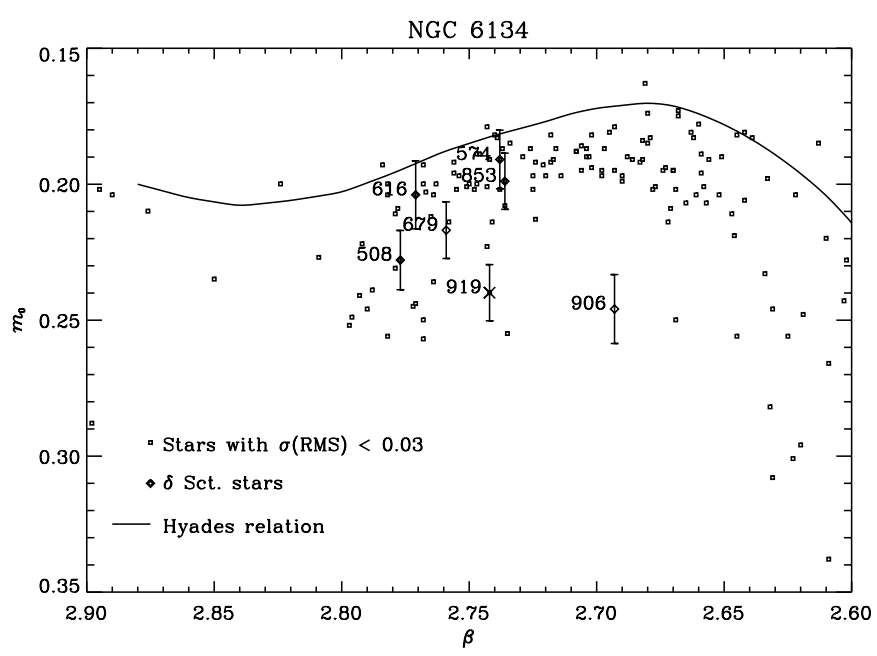

Fig. 5. The relation between $m_{0}$ index and $\beta$ for stars in NGC 6134 . The solid line is the Hyades relation adapted from Crawford (1975a, 1979). The error bars on $m_{0}$ are shown for the $\delta$ Scuti stars and the metal rich non-variable star \#919; the plotted errors include a systematic error of $0.02 \mathrm{mag}$. Note that only stars with photometric errors on $m_{0}$ and $\beta$ below 0.03 mag are shown.

Examples of the result of the automatic fit of abundances are shown in Fig. 4 for a Ca-line and five Fe-lines as seen in the spectrum of the $\delta$ Scuti star \#574. This star has quite low $v \sin i$, hence it is possible to find a number of Fe-lines that are only partially blended.
The abundances determined with vwa from the $\mathrm{Fe}$ and $\mathrm{Ca}$ lines, are presented in Table 5. The abundance of element $X$ is given as $\log N_{X} / N_{\text {tot }}$ where $N_{X}$ is the number of atoms of element $X$ and $N_{\text {tot }}$ is the total number of atoms in the atmosphere model. The error quoted in parenthesis in Table 5 is the RMS internal error.

To get a realistic idea of the error on the abundances one must consider the uncertainty of the parameters used for the computation of the model atmosphere and the synthetic spectrum, most noticeably $T_{\text {eff }}$ and microturbulence. We assume that a realistic error estimate of $T_{\text {eff }}$ is $200 \mathrm{~K}$ which corresponds to an error of about $0.1 \mathrm{dex}$ in the Fe abundance. For the $\delta$ Scuti stars we have used microturbulence in the range 1.4$2.5 \mathrm{~km} \mathrm{~s}^{-1}$. We estimate the uncertainty to be around $0.6 \mathrm{~km} \mathrm{~s}^{-1}$ which corresponds to an error in the Fe abundance of about 0.1 dex. For the final result we have combined the uncertainties due to the internal scatter (sd. of the mean) and the contributions from the estimated error on $T_{\text {eff }}$ and microturbulence.

The derived metallicities from photometry and spectroscopy are given in the last two columns of Table 5. The quoted errors include a $10 \mathrm{mmag}$ zero point error in the $m_{1}$ index (for $[\mathrm{Fe} / \mathrm{H}]_{\text {phot }}$ ) and uncertain model parameters (for $[\mathrm{Fe} / \mathrm{H}]_{\text {spec }}$ ) as was discussed above.

Abundance analysis was possible for four $\delta$ Scuti stars in NGC 6134 and the mean Fe abundance is $\log N_{\mathrm{Fe}} / N_{\text {tot }}=$ $-4.16 \pm 0.05$ (the quoted error is the sd. of the mean). Assuming that these stars have the same $\mathrm{H}$ and $\mathrm{He}$ abundances as the Sun we estimate the average metallicity of A-type stars in 
Table 5. Derived abundances for four $\delta$ Scuti stars stars and the twin star \#919 in NGC 6134, reference star HD 118646, and the Sun (from Grevesse et al. 1996). The first column is the identification number used by Bruntt et al. (1999). The numbers in parenthesis give the internal rms error and $n$ is the number of lines used. The microturbulence that was used for the computation of the synthetic lines is given - the approximate error is $0.6 \mathrm{~km} \mathrm{~s}^{-1}$. The last columns give the metallicity determined by the $m_{1}$ index and spectroscopy.

\begin{tabular}{lllllclll}
\hline \hline $\mathrm{ID}_{3}$ & $\log N_{\mathrm{Fe}} / N_{\text {tot }}$ & $n$ & $\log N_{\mathrm{Ca}} / N_{\text {tot }}$ & $n$ & $v_{\text {micro }}\left[\mathrm{km} \mathrm{s}^{-1}\right]$ & $m_{1}$ & {$[\mathrm{Fe} / \mathrm{H}]_{\text {phot }}$} & {$[\mathrm{Fe} / \mathrm{H}]_{\text {spec }}$} \\
\hline $508^{a}$ & $-4.05(0.21)$ & 16 & $-5.38(0.24)$ & 6 & 2.3 & 0.190 & $0.59(0.11)$ & $0.49(0.15)$ \\
574 & $-4.30(0.17)$ & 12 & $-4.99(0.49)$ & 7 & 2.5 & 0.104 & $0.23(0.11)$ & $0.24(0.15)$ \\
679 & $-4.16(0.27)$ & 7 & $-4.68(0.39)$ & 2 & 2.3 & 0.144 & $0.46(0.11)$ & $0.38(0.17)$ \\
906 & $-4.12(0.34)$ & 10 & $-4.65(0.09)$ & 4 & 1.4 & 0.181 & $1.01(0.15)$ & $0.42(0.18)$ \\
$919^{a}$ & $-4.09(0.28)$ & 11 & $-5.54(0.46)$ & 5 & 2.0 & 0.173 & $0.90(0.12)$ & $0.45(0.16)$ \\
$\mathrm{HD} 118646$ & $-4.44(0.20)$ & 12 & $-5.32(0.17)$ & 9 & 1.2 & - & $0.00(0.09)$ & $0.10(0.15)$ \\
Sun & -4.54 & - & -5.68 & - & - & - & 0.00 & - \\
\hline
\end{tabular}

${ }^{a}$ Possibly an Am star due to high $[\mathrm{Fe} / \mathrm{H}]$ and low $[\mathrm{Ca} / \mathrm{Fe}]$, see Conti (1970).

NGC 6134 to be $[\mathrm{Fe} / \mathrm{H}]_{\text {spec }}=+0.38 \pm 0.05$. This is in good agreement with the result from Strömgren photometry (F-type stars) which is $[\mathrm{Fe} / \mathrm{H}]_{\text {phot }}=+0.28 \pm 0.10$.

The $\delta$ Scuti stars \#508, \#906, and the twin star \#919 are found to be metal rich from the Strömgren photometry and this is confirmed by the spectroscopic results. For the stars \#508 and \#919 we find a low $[\mathrm{Ca} / \mathrm{Fe}]$ ratio which is characteristic for Am-type stars. We must stress that only few usable Ca lines could be found for abundance analysis, and hence the abundance is quite uncertain. Still, the result is interesting since our results indicate that the $\delta$ Scuti star \#508 is then possibly an Am-type star and at the same time a $\delta$ Scuti star.

\section{7. $\Delta$ a-photometry}

In this section we present results for $\Delta a$-photometry in order to shed more light on the true nature of the bona-fide Amtype candidates and the variable objects within NGC 6134. The $\Delta a$-system is capable to detect chemically peculiar stars (most efficient for magnetic CP2 stars in the notation of Preston 1974) via three narrow-band filter photometry. It samples the broad band flux depression around $520 \mathrm{~nm}$ in three filters: $g_{1}$ at $500 \mathrm{~nm}$ and $y$ at $550 \mathrm{~nm}$, both representing the continuum at the edges of the feature, and $g_{2}$ located at the center of the depression at $521 \mathrm{~nm}$. The $\Delta a$ magnitude is the difference of $g_{2}$ relative to the mean of $g_{1}$ and $y$, normalized to zero for a non-peculiar object: $a=g_{2}-\left(g_{1}+y\right) / 2$. The photometric accuracy needed to detect classical peculiar stars is typically a few mmags (Maitzen 1976). An $a$ versus $\left(g_{1}-y\right)$ diagram can therefore be used to detect peculiar objects and to sort out foreand background stars due to a high sensitivity to interstellar reddening. The standard relations are

$E\left(g_{1}-y\right)=0.54 \cdot E(b-y)$

$f=E(a) / E(b-y)$

with $f$ being of the order of 0.05 . If an open cluster does not suffer from differential reddening (such as NGC 6134), the normality line has a very small intrinsic scatter. Objects with a significant different reddening than the mean of the cluster, will be shifted almost horizontally in the $a$ versus $\left(g_{1}-y\right)$ diagram (see for example the case of IC 2602 in Maitzen 1993).

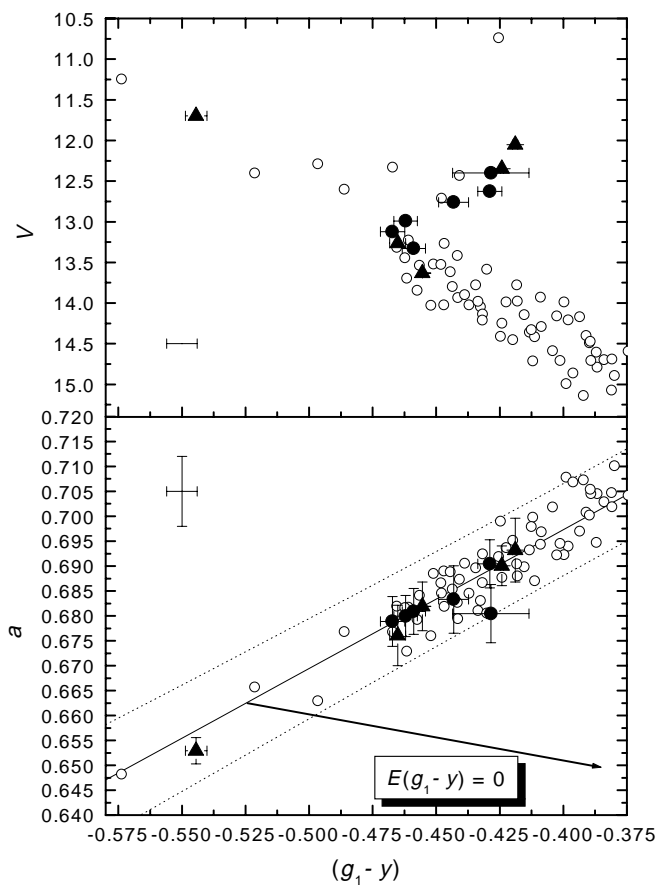

Fig. 6. $V$ and $a$ versus $\left(g_{1}-y\right)$ diagrams for NGC 6134 with the reddening vector for the temperature range from $\mathrm{A} 0$ to $\mathrm{F} 5$. Filled circles indicate bona-fide Am-type candidates whereas filled triangles are known variable stars from the literature. The full line is the normality line whereas the dotted lines are the confidence intervals corresponding to 99.9 percent. The error bars are shown for selected individual stars and the mean error bars are shown to the left.

What is the behaviour of Am-type stars in the $\Delta a$ photometric system? Maitzen (1976) and Vogt et al. (1998) investigated a sample of these objects. They found that the mean $\Delta a$-values are 6 mmag above the normality line with some very peculiar Am-type stars reaching $14 \mathrm{mmag}$. On the other hand classical CP stars have $\Delta a$ of up to $80 \mathrm{mmag}$.

The measurements for NGC 6134 are presented in Table 6. The errors on the variable stars are unfortunately too large to measure a $\Delta a \geq 0.006$ mmag. 
Table 6. The results of the $\Delta a$-photometry for bona-fide Am-type candidates (upper panel) and known variable stars (lower panel).

\begin{tabular}{crrccccccc}
\hline \hline $\mathrm{ID}_{1}$ & \multicolumn{1}{c}{$\mathrm{ID}_{2}$} & \multicolumn{1}{c}{$\mathrm{ID}_{3}$} & $V$ & $a$ & $\left(g_{1}-y\right)$ & $\Delta a$ & $(b-y)$ & $\Delta a$ & $N$ \\
\hline 5 & 106 & 832 & 12.400 & $0.680(6)$ & $-0.428(15)$ & -0.009 & 0.452 & -0.008 & 5 \\
23 & 50 & 1023 & 12.762 & $0.683(7)$ & $-0.443(6)$ & -0.002 & 0.434 & -0.002 & 30 \\
27 & 61 & 598 & 12.992 & $0.680(4)$ & $-0.462(5)$ & +0.000 & 0.423 & -0.004 & 32 \\
48 & 9 & 689 & 13.122 & $0.679(5)$ & $-0.467(5)$ & +0.000 & 0.417 & -0.004 & 32 \\
62 & 4 & 619 & 13.327 & $0.681(5)$ & $-0.459(5)$ & +0.000 & 0.422 & -0.003 & 32 \\
75 & 41 & 919 & 12.628 & $0.690(5)$ & $-0.429(5)$ & +0.001 & 0.460 & +0.001 & 30 \\
9 & 54 & 906 & 12.347 & $0.690(4)$ & $-0.424(4)$ & +0.000 & 0.486 & -0.003 & 30 \\
10 & 109 & 647 & 11.697 & $0.653(3)$ & $-0.544(3)$ & -0.004 & 0.262 & -0.006 & 30 \\
22 & 59 & 679 & 13.631 & $0.682(5)$ & $-0.455(5)$ & +0.000 & 0.424 & -0.002 & 32 \\
38 & 66 & & 13.264 & $0.676(6)$ & $-0.465(6)$ & -0.003 & & & 32 \\
57 & 15 & 853 & 12.051 & $0.693(6)$ & $-0.419(6)$ & +0.001 & 0.477 & +0.001 & 30 \\
85 & 21 & 735 & 14.452 & $0.695(5)$ & $-0.420(4)$ & +0.003 & 0.483 & +0.002 & 28 \\
\hline
\end{tabular}

Column 1: Identification from Paunzen \& Maitzen (2002).

Column 2: Identification from Lindoff (1972).

Column 3: Identification from Bruntt et al. (1999).

Column 4: Visual magnitude.

Columns 5, 6: mean $a$-index and its standard deviation.

Columns 7, 8: mean $\left(g_{1}-y\right)$ value and its standard deviation.

Column 9: Deviation from cluster line $(\Delta a)$.

Column 10: $(b-y)$ from the literature.

Column 11: Deviation from cluster line $(\Delta a)$.

Column 12: Number of measurements.

The normality lines based on both $(b-y)$ and $\left(g_{1}-y\right)$ have been determined from the observed sample of stars:

$a_{0}=0.618(3)+0.155(5) \cdot(b-y)$

and

$a_{0}=0.809(2)+0.279(6) \cdot\left(g_{1}-y\right)$.

The zero point of these relations are shifted less than $1 \mathrm{mmag}$ compared to relations derived for less metal rich clusters. Likewise, the slopes we have found here or similar to what we have found for the other clusters we have studied (Paunzen \& Maitzen 2002). The $3 \sigma$ detection limit was determined to be 9 mmag.

This limit is quite high and will not allow us to detect weak Am-type stars. The reason for the "high" detection limit at 9 mmag is that the normality line is not known with sufficient accuracy due to the combination of the photometric accuracy, the spread in metallicity and intrinsic chemical peculiarity, and possible non-uniform reddening across the field of NGC 6134.

From the correlation of 84 stars in common with Bruntt et al. (1999) we find the transformation of our $y$ measurements to standard $V$ values as:

$V=-8.138(12)+y$.

Figure 6 shows the $V$ and $a$ versus $\left(g_{1}-y\right)$ diagrams. The filled circles are the measured bona-fide Am-type candidates, i.e. star number 598, 619, 832, 919, and 1023, whereas the filled triangles are the known variable stars from the literature: star number 66 (number according to Lindoff 1972), 647, 679, 853, and 906. The lower panel of Fig. 6 includes the reddening vector corresponding to the mean of the cluster $E(b-y)=0.263 \mathrm{mag}$ and the relations 6 and 7. Table 6 summarizes the results of all relevant objects. From Fig. 6 we may conclude the following about the investigated objects (cf. Table 6):

- they are true members of NGC 6134;

- they do not show any significant enhancement of the $520 \mathrm{~nm}$ depression typical mainly for classical magnetic CP stars.

We are not able to definitely rule out an Am-type nature but it seems rather unlikely since all objects exhibit almost zero or even slightly negative $\Delta a$-values.

Five additional variables have been identified during the $\Delta a$-photometry with rather high level of variability compared to the $\delta$ Scuti stars discussed here. The identification numbers are 3 (number from Lindoff 1972), 735, 1046, 1115, and 1154 (the last four star numbers according to Bruntt et al. 1999). Due to the distribution of the observations, no conclusion about the nature of variability can be drawn. In the relevant temperature range one might think of $\delta$ Scuti and $\gamma$ Doradus pulsation as well as variability due to eclipsing binaries. Further photometric observations are needed to clarify the true nature of the variability of these five stars.

\section{The variables one by one}

Below we will describe the stars one by one. The detailed information on the known oscillation modes can be found in Viskum et al. (1996).

\subsection{Star \#508}

This star is one of two faint $\delta$ Scuti stars close to the main sequence. It is thus a bit surprising to find this star rotating slower than all other $\delta$ Scuti stars in the cluster, but it might of course 
be due to the inclination $i$ being close to zero. Two modes were detected with low amplitudes at $\sim 1.5 \mathrm{mmag}$. The result from Strömgren photometry and spectroscopy say that the star has a high $[\mathrm{Fe} / \mathrm{H}]$ (cf. Table 5). The $v \sin i=33 \pm 8$ is derived from six spectra. Due to the high metallicity $\mathrm{H} \beta$ was too contaminated to be used and the rotation is measured using only the $\mathrm{H} \alpha$ line. The star could very well be a marginal Am star. Its $[\mathrm{Fe} / \mathrm{H}]$ value is increased, but its $[\mathrm{Ca} / \mathrm{H}]$ value is lower than the mean for the other stars. This is the characteristics for Am-type stars (see Wolff 1983, Chap. 5). It might be a star similar to HD 1097: an Am-type star which is pulsating with low amplitudes and was discovered by Kurtz $(1989,2000)$. Unfortunately $\Delta a$ photometry was not made for this star.

\subsection{Star \#574}

This star is slightly evolved. Only a short time string was observed and only two modes could be detected with the coarse frequency resolution. The star rotates with a modest $v \sin i=$ $60 \pm 5$. Four spectra were analysed and both $\mathrm{H} \alpha$ and $\mathrm{H} \beta$ lines were used. The metallicity is similar to the cluster mean as determined from spectroscopy and photometry.

\subsection{Star \#616}

Both $\mathrm{H} \alpha$ and $\mathrm{H} \beta$ could be used in this star, but only one spectrum was observed. The lines are broad leading to the highest $v \sin i=163 \pm 14$ among the $\delta$ Scuti stars in NGC 6134. The star is only slightly evolved and is located exactly at the turnoff point of the cluster. Three modes have been identified, but more modes are probably present. The $m_{1}$ index is similar to the cluster mean, but an abundance analysis could not be made because of the high $v \sin i$ and the low signal to noise.

\subsection{Star \#679}

This is the second main sequence star with three modes identified at low amplitudes at 1-2 mmag. Five spectra were analysed and they indicate that the star is a fast rotator $v \sin i=145 \pm 13$ using the $\mathrm{H} \alpha$ line. The $S / N$ was good when combining the five spectra, so we could make an abundance analysis, but the $v \sin i$ is very high for this star so it is difficult to find good lines. Still, the derived metallicity agrees with the $m_{1}$ index, i.e. the star has slightly higher metallicity compared to the cluster mean.

\subsection{Star \#853}

This is the most evolved $\delta$ Scuti star with only a single relatively high amplitude mode identified with $A=6.2 \mathrm{mmag}$. It is rotating at $v \sin i=112 \pm 9$ which is derived from both the $\mathrm{H} \alpha$ and $\mathrm{H} \beta$ lines. The $m_{1}$ index is higher than the mean for the cluster, but we could not confirm this from the spectra of the star, since the $S / N$ was too low.

\subsection{Star \#906}

This is also an evolved star with five low frequency modes in the range $60-90 \mu \mathrm{Hz}$, where four modes are very closely spaced within $6 \mu \mathrm{Hz}$. The interpretation of the close frequencies as rotationally split modes is supported by the small $v \sin i=50 \pm 7$, mainly derived from $\mathrm{H} \alpha$. A high value of the $m_{1}$ index suggest that the star has a high metallicity, and the spectroscopic value for $[\mathrm{Fe} / \mathrm{H}]$ is in agreement, but showing a more modest value. It does not seem to be an Am-type star as the $\Delta a$ index and the spectroscopic $\mathrm{Ca}$ abundance do not show any deviation from the cluster mean.

\subsection{Star \#919}

The star is not a variable, but we include it here since it may well be an Am-type star: the $[\mathrm{Fe} / \mathrm{H}]$ is high, but it has a low $[\mathrm{Ca} / \mathrm{Fe}]$ ratio. The $\Delta a$ observations do not show indications of the star being an Am-type star, however. The projected rotational velocity is $v \sin i=88 \pm 6$.

\section{Discussion}

We have found that $\delta$ Scuti stars do not seem to be different from their twin stars in NGC 6134 in terms of their stellar parameters. This conclusion is consistent with Solano \& Fernley (1997) who used the same methods of calculation. Their study did show a difference in the spread of $v \sin i$ between low amplitude variables and non-variable stars, but the rather small number of stars in our study may be the cause of this difference.

What do we find in other open clusters? We have collected data similar to what we have for NGC 6134 from the open cluster database (Mermilliod 1996) and from the catalogue of rotational velocities for four nearby clusters (Glebocki \& Stawikowski 2000). Stars for which $v \sin i$ and Strömgren indices are available were chosen, and from this list stars with $0.09<(b-y)<0.23$ were selected, i.e. the A-type stars (the interstellar reddening is negligible for the nearby clusters examined here). The number of stars satisfying these criteria is small. The number of stars in each of the four open cluster lies in the range $14 \leq N \leq 31$.

We want to investigate if the distribution of $v \sin i$ for the $\delta$ Scuti stars is similar to non-variable stars in several open clusters. Unfortunately, the question is difficult to answer due to the small number of stars. Looking at Fig. 7 no overall pattern can be seen. In Praesepe the $\delta$ Scuti stars seem to be found almost exclusively among the fast rotators, but in the Pleiades the slow rotators are variable stars as well. In the remaining clusters including NGC 6134 the $\delta$ Scuti stars seem not to be restricted to any particular range of $v \sin i$.

The median of the distributions are presented in Table 7. There is clearly an indication that stars in the nearest clusters on average rotate faster than in $\alpha$ Per and NGC 6134.

Another aim of the present work is to investigate if there is any difference in the abundances of $\delta$ Scuti stars and nonvariable stars. We find that the $\delta$ Scuti stars generally seem to have metallicities similar to the cluster mean, although one of the $\delta$ Scuti stars may be a marginal Am-type star. Spectroscopy with higher resolution is needed to confirm these results. If slowly rotating stars can also be variable, it is not evident that the Am-type character prevents a star from pulsating. Indeed, it is possible that the $\kappa$-mechanism has not been blocked 


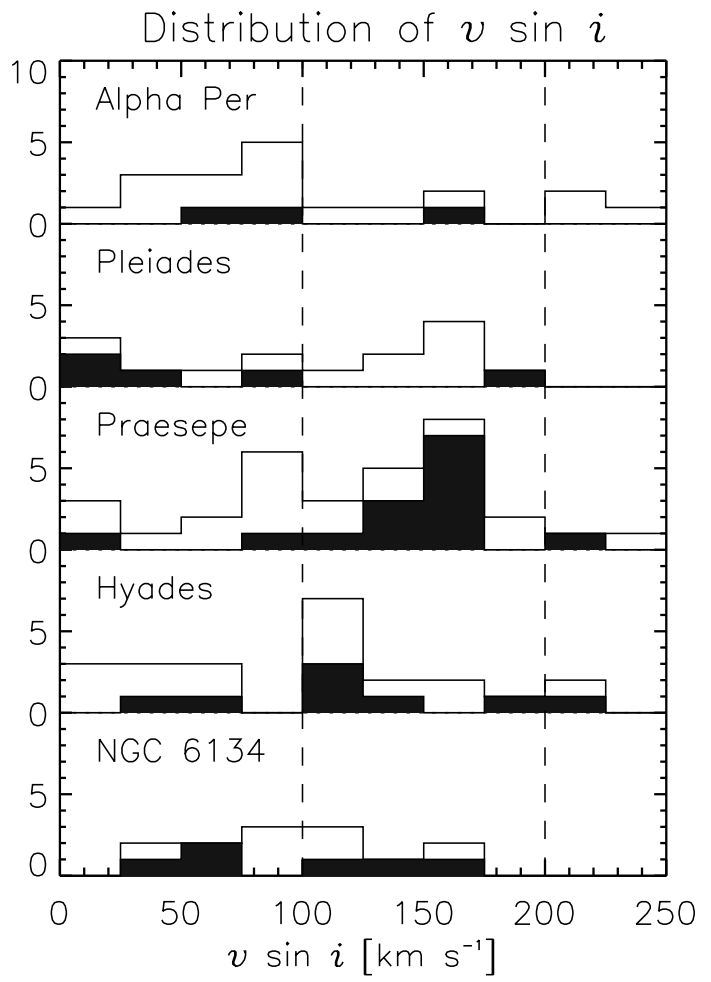

Fig. 7. Histograms of the distribution of rotational velocities in five open clusters. The black columns is the fraction of $\delta$ Scuti stars in a given column, e.g. in Hyades 3 of 7 stars in the range $100<v \sin i<$ $125 \mathrm{~km} \mathrm{~s}^{-1}$ are $\delta$ Scuti stars.

Table 7. Median of the distribution of $v \sin i$ for the whole cluster and the $\delta$ Scuti stars for five open clusters.

\begin{tabular}{lcc}
\hline \hline & \multicolumn{2}{c}{$v \sin i\left[\mathrm{~km} \mathrm{~s}^{-1}\right]$} \\
\hline Cluster & $\begin{array}{c}\text { Cluster } \\
\text { stars }\end{array}$ & $\begin{array}{c}\delta \text { Scuti } \\
\text { stars }\end{array}$ \\
\hline$\alpha$ Per & 85 & 75 \\
Pleiades & 115 & 25 \\
Praesepe & 130 & 152 \\
Hyades & 106 & 109 \\
NGC 6134 & 90 & 112 \\
\hline
\end{tabular}

by diffusion of He from the driving region in all Am-type or marginal Am-type stars.

\section{Conclusions}

We have presented the results of our analysis of spectra of variable and non-variable stars in the metal rich open cluster NGC 6134. We have determined the rotational velocity of several stars and made abundance analysis of the $\delta$ Scuti stars stars. We summarize our conclusions below:

- We have determined $v \sin i$ for the six known $\delta$ Scuti stars and a set of known non-variable A-type stars in NGC 6134.

- We have determined $[\mathrm{Fe} / \mathrm{H}]$ from spectroscopy and find a good agreement with the value from the $m_{1}$ index. We confirm that NGC 6134 is quite metal rich with $[\mathrm{Fe} / \mathrm{H}]$ around $0.3 \pm 0.1$ (error estimate includes systematic errors). From the $m_{1}$ index some stars seem to have enhanced atmospheric metal content and could be Am-type stars. We find that one of the $\delta$ Scuti stars seem to be a marginal Amtype star (star \#508).

- We have presented $\Delta a$ photometry which was carried out in order to detect any chemically peculiar stars. We find no classical Am-type stars in our sample of stars but we note that we did not obtain $\Delta a$ for the interesting $\delta$ Scuti star \#508.

- The distributions of rotational velocities of $\delta$ Scuti stars and non-variable A-stars in five well studied open clusters have been analysed: we find that the distributions are quite different but no safe conclusions can be drawn due to the small number of stars. For NGC 6134 we find that half of the known $\delta$ Scuti stars have low $v \sin i$ and they would be suitable for modeling by the use of asteroseismology in the future

Acknowledgements. We thank Werner W. Weiss (Vienna) for providing us with the grid of synthetic spectra. We are grateful for the Danish ESA Science Program (ESA Følgeforskning) grant No. 9902474.

\section{References}

Bayer, C., Maitzen, H. M., Paunzen, E., Rode-Paunzen, M., \& Sperl, M. 2000, A\&AS, 147, 99

Bruntt, H., Frandsen, S., Kjeldsen, H., \& Andersen, M. I. 1999, A\&AS, 140, 135

Bruntt, H., Catala, C., Garrido, R., et al. 2002, A\&A, 389, 345

Conti, P. S. 1970, PASP, 82, 781

Frandsen, S., Balona, L. A., Viskum, M., Koen, C., \& Kjeldsen, H. 1996, A\&A, 308, 132

Glebocki, R., \& Stawikowski, A. 2000, Acta Astron., 50, 509

Kjeldsen, H., Arentoft, T., Bedding, T., et al. 1998, in Proc. SOHO 6/GONG 98 Workshop, ed. S. G. Korzennik, \& A. Wilson (ESA Publications Division), 385

Kupka, F. 1996, ASP Conf. Ser. 108, ed. S. J Adelman, F. Kupka, \& W. W. Weiss, 73

Kupka, F., Piskunov, N., Ryabchikova, T. A., Stempels, H. C., \& Weiss, W. W. 1999, A\&AS, 138, 119

Kurtz, D. W. 1989, MNRAS, 238, 1077

Kurtz, D. W. 2000, in Delta Scuti and Related Stars, ed. M. Breger, \& M. H. Montgomery, ASP Conf. Ser., 210, 287

Kurucz, R. L. 1979, ApJS, 40, 1

Lindoff, U. 1972, A\&AS, 7, 231

Maitzen, H. M. 1976, A\&A, 51, 223

Maitzen, H. M., \& Vogt, N. 1983, A\&A, 123, 48

Maitzen, H. M. 1985, A\&AS, 62, 129

Maitzen, H. M. 1993, A\&AS, 102, 1

Pérez Hernández, F., Claret, A., Hernández, M. M., \& Michel, E. 1999, A\&A, 346, 586

Mermilliod, J.-C. 1996, ASP Conf. Ser., 90, ed. E. F. Milone, \& J.-C. Mermilliod (see WEBDA open cluster database: http://obswww. unige.ch/webda/), 475

Paunzen, E., \& Maitzen, H. M. 2002, A\&A, submitted

Rodríguez, E., López-González, M. J., \& López de Coca, P. 2000, A\&AS, 144, 469

Rogers, N. Y. 1995, Comm. Asteroseismol., 78

Smalley, B. 1993, A\&A, 274, 391

Solano, E., \& Fernley, J. 1997, A\&AS, 122, 131

Stetson, P. B. 1983, AJ, 88, 1349

Vogt, N., Kerschbaum, F., Maitzen, H. M., \& Faúndez-Abans, M. 1998, A\&AS, 130, 455

Wolff, S. C. 1983, The A-stars: problems and perspectives, NASA SP-463 